\title{
20. EOCENE-MIOCENE MAGNETOSTRATIGRAPHY OF THE SOUTHEAST GREENLAND MARGIN AND WESTERN IRMINGER BASIN ${ }^{1}$
}

\author{
Jason R. Ali² and Didier Vandamme
}

\begin{abstract}
Shore-based paleomagnetic investigations were conducted on Eocene-Miocene sediments recovered during Ocean Drilling Program Leg 152 drilling of the southeast Greenland Margin and the western Irminger Basin. A total of 147 specimens was analyzed. In general, the magnetostratigraphy obtained from the shipboard whole-core magnetometer studies (spot demagnetization at $25 \mathrm{mT}$ ) has been confirmed. However, a number of specimens yielded polarities different from those obtained by the shipboard magnetometer, calling for some caution in the interpretation of the magnetostratigraphy. During Leg 152, it proved difficult to correlate the polarity records with the geomagnetic polarity time scale because of core recovery and the limited biostratigraphic information available at the time. Since then, the shore-based biostratigraphic studies have yielded many datum points useful for correlating the refined magnetozone sequences to the polarity time scale. In terms of seaward dipping reflector formation, the new data are particularly valuable in determining their subsidence history. At Sites 915 and 917 , close to the southeast Greenland ocean/continent transition, intervals of $13 \mathrm{~m} . \mathrm{y}$. and $4 \mathrm{~m} . \mathrm{y}$. ., respectively, elapsed before marine sedimentation commenced. Sedimentation at Site 918, in the western part of the Irminger Basin, commenced almost immediately after seaward dipping reflector formation at about $54 \mathrm{Ma}$.
\end{abstract}

\section{INTRODUCTION}

In this paper, we present the results of shore-based magnetostratigraphic studies of Eocene-Miocene sediments from Ocean Drilling Program (ODP) Leg 152. To varying degrees, our results are relevant to all of the cruise objectives: formation of the southeast Greenland seaward dipping reflector sequences (SDRS) during Paleocene-earliest Eocene times, Northern Hemisphere glaciation (late MioceneHolocene), Neogene Arctic-Atlantic paleoceanography and North Atlantic Eocene-Oligocene chronostratigraphy. The drilling at all six of the sites (914-919) penetrated Cenozoic sediments overlying volcanic basement (SDRS) of presumed C24r age (except Site 919, C24n.3n age); see also Larsen and Saunders (this volume).

\section{LABORATORY PROCEDURES}

A full account of the shipboard paleomagnetic laboratory procedures is provided in Larsen, Saunders, Clift, et al. (1994). In summary, 1.5-m-long archive-half sections were analyzed for their paleomagnetism using a $2 \mathrm{G}$ Enterprises cryogenic magnetometer with an in-line, three-axis alternating field (AF) demagnetization system. The $\mathrm{AF}$ system is capable of generating fields of up to $35 \mathrm{mT}$. Prior to AF cleaning, the initial remanent magnetization of the cores was measured at $10-\mathrm{cm}$ intervals along each section. Unfortunately, because of the large volumes of material that had to be analyzed aboard ship, only one demagnetization $(25 \mathrm{mT})$ and one measurement cycle could be performed on each section. This limited us in fully investigating the nature and origin of the natural remanent magnetization (NRM) within the rocks examined.

To enhance the reliability of the polarity record defined from shipboard measurements, detailed post-cruise shore-based studies were

${ }^{1}$ Saunders, A.D., Larsen, H.C., and Wise, S.W., Jr. (Eds.), 1998. Proc. ODP, Sci. Results, 152: College Station, TX (Ocean Drilling Program).

${ }^{2}$ Department of Oceanography, The University, Southampton, SO17 1BJ, United Kingdom.

${ }^{3}$ J.E. Geochimie et Magnetisme des Roches, Universite d'Aix-Marseille III, 13397, Marseille Cedex 20, France. performed. Typically, one sample per core section was taken for these studies. Samples were measured using a $2 \mathrm{G}$ Enterprises cryogenic magnetometer, with an in-line AF demagnetizing system capable of generating fields of up to $40 \mathrm{mT}$. After initial measurement of the NRM, samples were demagnetized at 5-mT step increments up to $40 \mathrm{mT}$ to isolate the primary remanence. Samples requiring additional demagnetization were processed to 50-70 mT using a Molspin tumbling AF system. In practically all cases, the magnetization comprises a single low-coercivity component (which generally is indicative of a simple magnetization history). Examples of the responses of samples to AF demagnetization are shown in Figure 1.

\section{BIOSTRATIGRAPHIC CONSTRAINTS AND GEOMAGNETIC POLARITY TIME SCALE}

During Leg 152 and in our report in Larsen, Saunders, Clift, et al. (1994), we used the Cande and Kent (1992) geomagnetic polarity time scale. In this paper, we use the Cande and Kent (1995) time scale.

The magnetostratigraphy obtained on JOIDES Resolution provided an apparently clear pattern of normal and reverse polarity magnetozones within the Eocene-Miocene succession. However, correlating this record with the geomagnetic polarity time scale (GPTS) proved somewhat difficult because of the limited recovery in certain intervals and because of the lack of age-diagnostic microfaunas at many levels. As far as Leg 152 is concerned, the nannoplankton are the most useful fossil group with which to link the obtained polarity record to the GPTS. A refined nannofossil stratigraphy has been provided by Wei (this volume). Additional information is provided by Jolley (this volume) on the Eocene palynomorphs.

\section{SITE 914}

At Site 914, only Cores 152-914B-15R through 17R recovered Eocene-Miocene sediments. Shipboard biostratigraphic studies assigned these three cores to Zones CP16-CP18, indicating an early Oligocene age. The magnetic polarity sequence obtained from the 
A

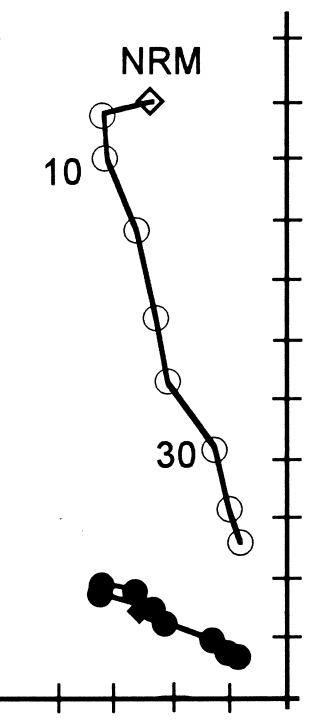

1 unit $=44.82 \mathrm{~mA} / \mathrm{m}$
B

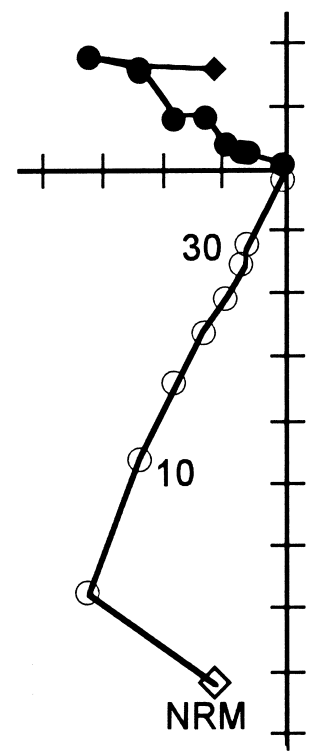

1 unit $=47.43 \mathrm{~mA} / \mathrm{m}$
Figure 1. Examples of responses to AF demagnetization. Data plotted on Zijderveld (1967) diagrams. Closed symbols represent the vectors plotted on the horizontal plane and open symbols represent the vectors plotted on a vertical plane. Numbers alongside both vertical plane plots indicate the applied demagnetization field (mT). A. Sample 152-918D-25R-3, 14 cm. B. Sample 152-918D-36R-1, $114 \mathrm{~cm}$.

cores was correlated with geomagnetic Chrons $\mathrm{C} 13 \mathrm{n}-\mathrm{C} 12 \mathrm{r}$ although a number of other, theoretically plausible, correlations are possible. Shore-based paleomagnetic studies were not performed on material from this site. New biostratigraphic information indicates a narrower age range for the three cores (CP16) in support of the C13n-12r magnetostratigraphic correlation. Accordingly, the age of these cores is around $33 \mathrm{Ma}$ (Cande and Kent, 1995).

\section{SITE 915}

At Site 915, only Cores 152-915A-11R through 23R recovered middle through upper Eocene sediments; no Oligocene-Miocene sediments were cored. Shipboard measurements were taken only on Cores $152-915 \mathrm{~A}-16 \mathrm{R}$ and $18 \mathrm{R}$ through $22 \mathrm{R}$, where the sediments were undisturbed. The polarity sequence obtained shipboard is relatively simple, with Core $152-915 \mathrm{~A}-16 \mathrm{R}$ carrying a normal polarity and Cores 152-915A-18R through $22 \mathrm{R}$ carrying a reverse polarity. Only slight modifications to the magnetostratigraphy are needed following shore-based work (Table 1). Sample 152-915A-16R-1, 102 $\mathrm{cm}$, carries a reverse polarity magnetization, contrary to the normal polarity previously identified at this level. Samples $152-915 \mathrm{~A}-18 \mathrm{R}-$ $1,61 \mathrm{~cm}, 21 \mathrm{R}-1,126 \mathrm{~cm}$, and 21R-2, $10 \mathrm{~cm}$, carry unstable magnetizations, and the reverse polarity record at these levels must be regarded as suspect. Reliable polarity data have also been obtained from three levels that were not investigated on the ship (Table 1). This new information confirms the record of a dominantly reverse polarity magnetization in Cores 152-915A-18R through 22R, which, according to the sparse biostratigraphic data, suggests a correlation with Chron C18r (middle Eocene) that is considerably younger than the volcanic basement.
Table 1. Summary of discrete specimen paleomagnetic data from Holes 915A, 916A, and 917A.

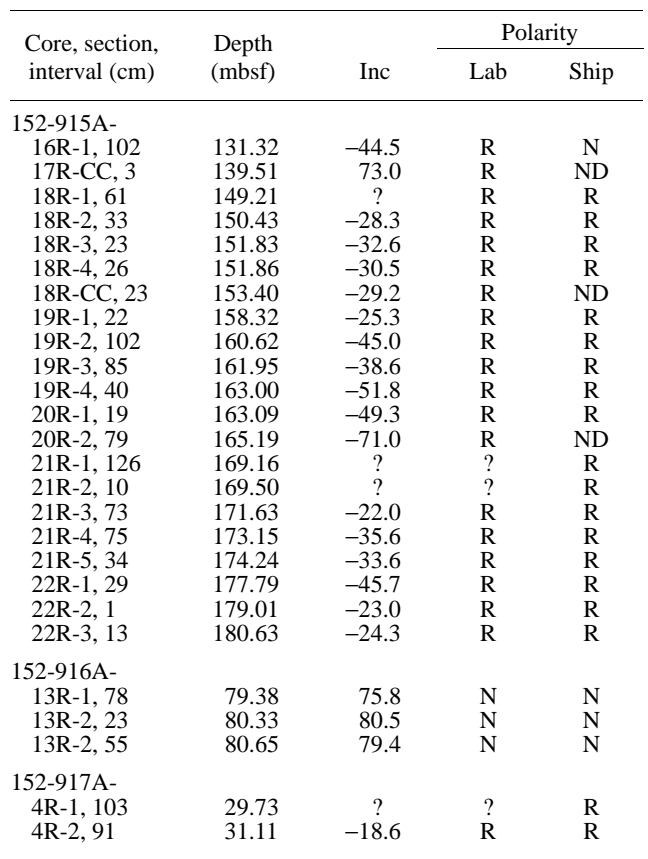

Notes: Inc $=$ characteristic remanent magnetization inclination. In Inc column, $?=$ no characteristic direction. Lab/Ship = polarity of equivalent levels from discrete samples analyzed onshore and shipboard whole-core determinations; $\mathrm{N}=$ normal, $\mathrm{R}=$ reverse, $?=$ indeterminate , and $\mathrm{ND}=$ no data.

\section{SITE 916}

Normal polarity data from sediments assumed from seismic stratigraphy to be of Eocene-Oligocene age were obtained from Cores 152-916A-13R and 14R, immediately above basement (Table 1). Unfortunately, shore-based biostratigraphic studies (Wei, this volume) have not yielded any fossil markers from this interval. Thus, it is not possible to correlate the magnetostratigraphy with the GPTS.

\section{SITE 917}

A single hole (917A) was drilled at this site through a 41.9-mthick, post-SDRS sedimentary cover. In the sediments, magnetic polarity data were obtained only from Core 152-917A-4R (reversely magnetized). Subsequent shore-based studies yielded a CP14a (middle Eocene) nannoplankton date for this core (Wei, this volume). Shore-based paleomagnetic studies were performed on two specimens (Table 1). Sample 152-917A-4R-1, $103 \mathrm{~cm}$, yielded an indeterminate polarity. Sample 152-917A-4R-2, $91 \mathrm{~cm}$, confirms the presence of a reverse polarity magnetization in this core. Linkage to Chron $\mathrm{C} 21 \mathrm{r}$ is the only feasible correlation for this magnetozone.

\section{SITE 918}

At Site 918, 120 specimens were analyzed from the $\sim 690-\mathrm{m}$ thick, Eocene-Miocene sequence drilled in Hole 918D. On the whole, there is good agreement between ship and shore-based polarity determinations, except at levels within Cores 152-918D-42R and 
Table 2. Summary of discrete specimen paleomagnetic data from Hole 918D.

\begin{tabular}{|c|c|c|c|c|c|c|c|c|c|}
\hline \multirow{2}{*}{$\begin{array}{l}\text { Core, section, } \\
\text { interval }(\mathrm{cm})\end{array}$} & \multirow{2}{*}{$\begin{array}{l}\text { Depth } \\
\text { (mbsf) }\end{array}$} & \multirow[b]{2}{*}{ Inc } & \multicolumn{2}{|c|}{ Polarity } & \multirow{2}{*}{$\begin{array}{l}\text { Core, section, } \\
\text { interval }(\mathrm{cm})\end{array}$} & \multirow{2}{*}{$\begin{array}{l}\text { Depth } \\
\text { (mbsf) }\end{array}$} & \multirow[b]{2}{*}{ Inc } & \multicolumn{2}{|c|}{ Polarity } \\
\hline & & & $\mathrm{Lab}$ & Ship & & & & $\mathrm{Lab}$ & Ship \\
\hline \multicolumn{10}{|l|}{ 152-918D- } \\
\hline $24 \mathrm{R}-1,35$ & 503.54 & 54.2 & $\mathrm{~N}$ & $\mathrm{~N}$ & $42 \mathrm{R}-3,105$ & 680.84 & 24.6 & $\mathrm{R}$ & $\mathrm{N}$ \\
\hline $24 \mathrm{R}-2,27$ & 504.96 & -62.7 & $\mathrm{R}$ & $\mathrm{R}$ & $42 \mathrm{R}-3,144$ & 681.23 & -38.4 & $\mathrm{R}$ & ? \\
\hline 24R-3, 21 & 506.21 & -50.8 & $\mathrm{R}$ & $\mathrm{R}$ & $42 \mathrm{R}-4,52$ & 681.82 & -47.0 & $\mathrm{R}$ & $\mathrm{R}$ \\
\hline $25 \mathrm{R}-1,81$ & 513.60 & -66.6 & $\mathrm{R}$ & $\mathrm{R}$ & $42 \mathrm{R}-4,131$ & 682.60 & -53.6 & $\mathrm{R}$ & $\mathrm{R}$ \\
\hline $25 \mathrm{R}-2,24$ & 514.03 & -46.7 & $\mathrm{R}$ & $\mathrm{R}$ & $42 \mathrm{R}-5,113$ & 683.92 & -64.1 & $\mathrm{R}$ & $\mathrm{R}$ \\
\hline $25 \mathrm{R}-3,14$ & 514.50 & -67.0 & $\mathrm{R}$ & $\mathrm{R}$ & 44R-1, 47 & 697.56 & 62.3 & $\mathrm{~N}$ & $\mathrm{~N}$ \\
\hline $25 \mathrm{R}-5,19$ & 517.12 & 64.8 & $\mathrm{~N}$ & $\mathrm{~N}$ & $44 \mathrm{R}-3,143$ & 700.52 & 62.9 & $\mathrm{~N}$ & $\mathrm{~N}$ \\
\hline $27 \mathrm{R}-1,30$ & 532.39 & -63.8 & $\mathrm{R}$ & $\mathrm{R}$ & $44 \mathrm{R}-4,52$ & 701.12 & 75.2 & $\mathrm{~N}$ & $\mathrm{~N}$ \\
\hline $27 \mathrm{R}-2,17$ & 533.76 & -63.6 & $\mathrm{R}$ & $\mathrm{R}$ & $44 \mathrm{R}-5,4$ & 702.50 & 55.0 & $\mathrm{~N}$ & $\mathrm{~N}$ \\
\hline $27 \mathrm{R}-3,30$ & 535.39 & -62.8 & $\mathrm{R}$ & $\mathrm{R}$ & $47 \mathrm{R}-2,55$ & 725.74 & -30.7 & $\mathrm{R}$ & $\mathrm{R}$ \\
\hline $28 \mathrm{R}-1,48$ & 542.27 & -63.7 & $\mathrm{R}$ & $\mathrm{R}$ & $47 \mathrm{R}-2,86$ & 726.05 & -70.9 & $\mathrm{R}$ & $\mathrm{N}$ \\
\hline $28 \mathrm{R}-2,81$ & 544.10 & 70.4 & $\mathrm{~N}$ & $\mathrm{~N}$ & $51 \mathrm{R}-1,83$ & 764.02 & -36.7 & $\mathrm{R}$ & $\mathrm{N}$ \\
\hline 28R-3, 37 & 545.16 & 70.3 & $\mathrm{~N}$ & $\mathrm{~N}$ & $51 \mathrm{R}-2,128$ & 765.97 & -42.1 & $\mathrm{R}$ & $\mathrm{R}$ \\
\hline $2 \mathrm{BR}-4,35$ & 546.64 & 67.4 & $\mathrm{~N}$ & $\mathrm{~N}$ & $51 \mathrm{R}-4,25$ & 767.95 & 72.5 & $\mathrm{~N}$ & $\mathrm{~N}$ \\
\hline $29 \mathrm{R}-1,58$ & 551.97 & 72.9 & $\mathrm{~N}$ & $\mathrm{~N}$ & $51 \mathrm{R}-5,145$ & 770.64 & 53.4 & $\mathrm{~N}$ & $\mathrm{~N}$ \\
\hline 29R-2, 36 & 553.25 & 67.0 & $\mathrm{~N}$ & $\mathrm{~N}$ & $52 \mathrm{R}-1,122$ & 774.11 & 53.4 & $\mathrm{~N}$ & $\mathrm{~N}$ \\
\hline 29R-CC, 13 & 554.16 & -71.7 & $\mathrm{R}$ & ND & $52 \mathrm{R}-3,58$ & 776.47 & 60.9 & $\mathrm{~N}$ & $\mathrm{~N}$ \\
\hline $31 \mathrm{R}-1,86$ & 571.55 & -57.1 & $\mathrm{R}$ & $\mathrm{R}$ & $52 \mathrm{R}-4,32$ & 777.71 & 65.2 & $\mathrm{~N}$ & $\mathrm{~N}$ \\
\hline $32 \mathrm{R}-1,98$ & 581.27 & 71.6 & $\mathrm{~N}$ & $\mathrm{~N}$ & $52 \mathrm{R}-5,80$ & 779.70 & -53.5 & $\mathrm{R}$ & $\mathrm{R}$ \\
\hline 33R-CC, 10 & 591.49 & 72.4 & $\mathrm{~N}$ & ND & $53 \mathrm{R}-1,35$ & 782.84 & 70.0 & $\mathrm{~N}$ & $\mathrm{~N}$ \\
\hline $34 \mathrm{R}-1,20$ & 599.79 & -52.0 & $\mathrm{R}$ & $\mathrm{R}$ & $53 \mathrm{R}-1,62$ & 783.11 & 68.4 & $\mathrm{~N}$ & $\mathrm{~N}$ \\
\hline $34 \mathrm{R}-1,106$ & 600.65 & 57.8 & $\mathrm{R}$ & $\mathrm{N}$ & $53 \mathrm{R}-1,116$ & 783.65 & 76.8 & $\mathrm{~N}$ & $\mathrm{~N}$ \\
\hline $34 \mathrm{R}-2,5$ & 601.14 & -52.1 & $\mathrm{R}$ & $\mathrm{R}$ & $53 \mathrm{R}-2,22$ & 784.21 & -31.8 & $\mathrm{R}$ & $\mathrm{R}$ \\
\hline $35 \mathrm{R}-1,18$ & 609.38 & 3.3 & ? & ? & $53 \mathrm{R}-2,134$ & 785.33 & 55.0 & $\mathrm{~N}$ & $\mathrm{~N}$ \\
\hline $36 \mathrm{R}-1,114$ & 620.03 & 60.4 & $\mathrm{~N}$ & $\mathrm{~N}$ & $53 \mathrm{R}-3,19$ & 785.68 & -43.9 & $\mathrm{R}$ & $\mathrm{R}$ \\
\hline 36R-CC, 3 & 622.83 & -66.0 & $\mathrm{R}$ & $\mathrm{R}$ & $53 \mathrm{R}-3,8$ & 785.87 & -42.4 & $\mathrm{R}$ & $\mathrm{R}$ \\
\hline $37 \mathrm{R}-1,132$ & 629.91 & -55.6 & $\mathrm{R}$ & $\mathrm{R}$ & $53 \mathrm{R}-3,10$ & 786.45 & -61.4 & $\mathrm{R}$ & $\mathrm{R}$ \\
\hline $37 \mathrm{R}-2,79$ & 630.88 & -50.9 & $\mathrm{R}$ & $\mathrm{R}$ & $53 \mathrm{R}-5,16$ & 789.48 & -48.4 & $\mathrm{R}$ & $\mathrm{R}$ \\
\hline $37 \mathrm{R}-2,121$ & 631.30 & 80.2 & $\mathrm{~N}$ & $\mathrm{R}$ & 53R-6, 41 & 790.92 & -49.1 & $\mathrm{R}$ & $\mathrm{R}$ \\
\hline 37R-3, 8 & 631.67 & -43.5 & $\mathrm{R}$ & $\mathrm{R}$ & $55 \mathrm{R}-1,35$ & 802.14 & 67.5 & $\mathrm{~N}$ & $\mathrm{~N}$ \\
\hline $37 \mathrm{R}-3,54$ & 632.13 & 74.6 & $\mathrm{~N}$ & $\mathrm{~N}$ & $55 \mathrm{R}-3,38$ & 805.17 & 76.4 & $\mathrm{~N}$ & $\mathrm{~N}$ \\
\hline $37 \mathrm{R}-4,23$ & 633.32 & 62.2 & $\mathrm{~N}$ & $\mathrm{~N}$ & $55 \mathrm{R}-5,16$ & 807.67 & 65.8 & $\mathrm{~N}$ & $\mathrm{R}$ \\
\hline $38 \mathrm{R}-2,127$ & 640.97 & 54.1 & $\mathrm{~N}$ & $\mathrm{~N}$ & $55 \mathrm{R}-6,41$ & 809.42 & 67.2 & $\mathrm{R}$ & $\mathrm{R}$ \\
\hline $38 \mathrm{R}-4,74$ & 643.43 & 80.7 & $\mathrm{~N}$ & $\mathrm{~N}$ & $57 \mathrm{R}-1,112$ & 822.11 & -55.1 & $\mathrm{R}$ & $\mathrm{R}$ \\
\hline $38 \mathrm{R}-6,19$ & 645.88 & 68.9 & $\mathrm{~N}$ & $\mathrm{~N}$ & $57 \mathrm{R}-2,134$ & 823.83 & $?$ & $\mathrm{R}$ & $\mathrm{R}$ \\
\hline $39 \mathrm{R}-1,27$ & 648.16 & $?$ & ? & $\mathrm{N}$ & $57 \mathrm{R}-3,144$ & 825.43 & -26.3 & $\mathrm{R}$ & $\mathrm{R}$ \\
\hline $39 \mathrm{R}-1,52$ & 648.41 & 84.0 & $\mathrm{~N}$ & $\mathrm{~N}$ & $57 \mathrm{R}-4,36$ & 825.85 & 69.1 & $\mathrm{~N}$ & $\mathrm{~N}$ \\
\hline $39 \mathrm{R}-1,96$ & 648.86 & $?$ & ? & $\mathrm{N}$ & $62 \mathrm{R}-1,7$ & 869.37 & $?$ & ? & ? \\
\hline $39 \mathrm{R}-1,137$ & 649.26 & 62.9 & $\mathrm{~N}$ & $\mathrm{~N}$ & $62 \mathrm{R}-2,30$ & 871.11 & 68.5 & $\mathrm{~N}$ & $\mathrm{~N}$ \\
\hline 39R-2, 18 & 649.57 & -44.6 & $\mathrm{R}$ & $\mathrm{R}$ & $63 \mathrm{R}-1,87$ & 879.76 & -19.4 & $\mathrm{R}$ & $\mathrm{N}$ \\
\hline $39 \mathrm{R}-2,123$ & 650.62 & -61.6 & $\mathrm{R}$ & $\mathrm{R}$ & $63 \mathrm{R}-2,32$ & 880.71 & -53.4 & $\mathrm{R}$ & $\mathrm{R}$ \\
\hline 39R-3, 11 & 651.00 & 70.1 & $\mathrm{~N}$ & $\mathrm{~N}$ & $74 \mathrm{R}-1,24$ & 982.93 & 69.1 & $\mathrm{~N}$ & $\mathrm{~N}$ \\
\hline 39R-3, 21 & 651.10 & $?$ & ? & $\mathrm{N}$ & $75 \mathrm{R}-1,39$ & 992.68 & -43.7 & $\mathrm{R}$ & $\mathrm{N}$ \\
\hline $39 \mathrm{R}-3,114$ & 652.03 & $?$ & ? & ? & $75 \mathrm{R}-2,52$ & 994.31 & 58.9 & $\mathrm{~N}$ & $\mathrm{~N}$ \\
\hline 39R-5, 14 & 654.03 & -61.2 & $\mathrm{R}$ & $\mathrm{R}$ & $75 \mathrm{R}-3,71$ & 996.00 & 47.3 & $\mathrm{~N}$ & $\mathrm{~N}$ \\
\hline $39 \mathrm{R}-6,64$ & 656.03 & -62.7 & $\mathrm{R}$ & $\mathrm{R}$ & $75 \mathrm{R}-4,26$ & 996.55 & 48.7 & $\mathrm{~N}$ & $\mathrm{~N}$ \\
\hline 40R-1, 46 & 657.95 & -52.5 & $\mathrm{R}$ & $\mathrm{R}$ & $76 \mathrm{R}-1,51$ & 1002.50 & -36.7 & $\mathrm{R}$ & $\mathrm{N}$ \\
\hline $40 \mathrm{R}-2,128$ & 660.27 & 52.1 & $\mathrm{R}$ & $\mathrm{R}$ & $77 \mathrm{R}-1,20$ & 1011.79 & 4.2 & ? & $\mathrm{N}$ \\
\hline $40 \mathrm{R}-4,91$ & 662.80 & 56.7 & $\mathrm{~N}$ & $\mathrm{~N}$ & $78 \mathrm{R}-1,21$ & 1021.40 & 65.0 & $\mathrm{~N}$ & $\mathrm{~N}$ \\
\hline $40 \mathrm{R}-6,11$ & 664.60 & 65.1 & $\mathrm{~N}$ & $\mathrm{~N}$ & 79R-1, 9 & 1030.98 & 77.1 & $\mathrm{~N}$ & $\mathrm{~N}$ \\
\hline $41 \mathrm{R}-1,92$ & 668.11 & -45.1 & $\mathrm{~N}$ & $\mathrm{~N}$ & $80 \mathrm{R}-1,27$ & 1040.86 & 38.5 & $\mathrm{R}$ & $\mathrm{N}$ \\
\hline 41R-3, 146 & 671.65 & 64.4 & $\mathrm{~N}$ & $\mathrm{~N}$ & $89 \mathrm{R}-1,15$ & 1127.54 & 46.9 & $\mathrm{~N}$ & $\mathrm{~N}$ \\
\hline 41R-5, 127 & 674.47 & 59.8 & $\mathrm{~N}$ & ND & $89 \mathrm{R}-4,31$ & 1131.70 & 59.1 & $\mathrm{R}$ & $\mathrm{R}$ \\
\hline 41R-CC, 24 & 674.94 & 73.7 & $\mathrm{~N}$ & $\mathrm{~N}$ & $90 \mathrm{R}-3,82$ & 1139.75 & -67.0 & $\mathrm{~N}$ & $\mathrm{~N}$ \\
\hline 42R-1, 72 & 677.51 & 73.9 & $\mathrm{~N}$ & $\mathrm{~N}$ & 93R-1, 24 & 1165.99 & 44.7 & $\mathrm{~N}$ & $\mathrm{~N}$ \\
\hline $42 \mathrm{R}-2,12$ & 678.41 & -25.3 & $\mathrm{R}$ & $\mathrm{N}$ & 93R-1, 135 & 1167.14 & 69.0 & $\mathrm{~N}$ & $\mathrm{~N}$ \\
\hline $42 \mathrm{R}-2,74$ & 679.04 & 29.0 & $\mathrm{~N}$ & $\mathrm{~N}$ & $95 \mathrm{R}-1,18$ & 1180.57 & -63.9 & $\mathrm{R}$ & $\mathrm{N}$ \\
\hline $42 \mathrm{R}-2,141$ & 679.70 & 44.0 & $\mathrm{R}$ & $\mathrm{R}$ & $95 \mathrm{R}-2,20$ & 1181.00 & -72.8 & $\mathrm{R}$ & $\mathrm{N}$ \\
\hline $42 \mathrm{R}-3,39$ & 680.18 & -35.9 & $\mathrm{R}$ & ? & $95 \mathrm{R}-3,74$ & 1183.03 & 39.3 & $\mathrm{~N}$ & $\mathrm{R}$ \\
\hline $42 \mathrm{R}-3,70$ & 680.49 & 43.0 & $\mathrm{R}$ & ? & 96R-1, 33 & 1185.42 & -25.8 & $\mathrm{R}$ & $\mathrm{R}$ \\
\hline
\end{tabular}

Notes: Inc $=$ characteristic remanent magnetization inclination. In Inc column, $?=$ no characteristic direction. Lab/Ship $=$ polarity of equivalent levels from discrete samples analyzed onshore and shipboard whole-core determinations; $\mathrm{N}=$ normal, $\mathrm{R}=$ reverse, ? = indeterminate, and $\mathrm{ND}=$ no data.

95R. (Table 2). Although core recovery averaged $\sim 35 \%$, two major intervals (703-763 meters below seafloor [mbsf] and 825-1127 mbsf) had almost negligible recovery, making correlation between magnetozones and the GPTS difficult (Fig. 2).

Nannoplankton studies have been conducted on more than 170 Eocene-Miocene specimens (Wei, this volume). Additionally, 25 samples from the Eocene succession have been examined for palynomorphs (Jolley, this volume).

The most reliable magnetobiostratigraphic information comes from the lower part of the sequence, between about 1118 and 1189 mbsf, where the biostratigraphic data permit reasonably sound corre- lations. The proposed correlations are: end $\mathrm{C} 24 \mathrm{r}$ at $1185 \mathrm{mbsf}$, C24n.3n at $1185-1180$ mbsf, C24n.1n at 1168-1166 mbsf, C23n at 1158-1156 mbsf, C22n-C20n at 1148-1127 mbsf, and C19n at 1119-1118 mbsf. Other possible correlations higher in the sequence are: Chrons C6n at $807-802$ mbsf, C5Dr-5Cn at 792-766 mbsf, C5AC/5AD or C5AC-AD at 703-696 mbsf, C5r at 658-651 mbsf, and $\mathrm{C} 3 \mathrm{r}$ at $516-505 \mathrm{mbsf}$.

Basement at Site 918 almost certainly formed during Chron C24r (Larsen and Saunders, this volume). Therefore, there is only a minimal time lag between SDRS formation and subsidence to a depth at which marine sedimentation commenced (Chron C24r). 


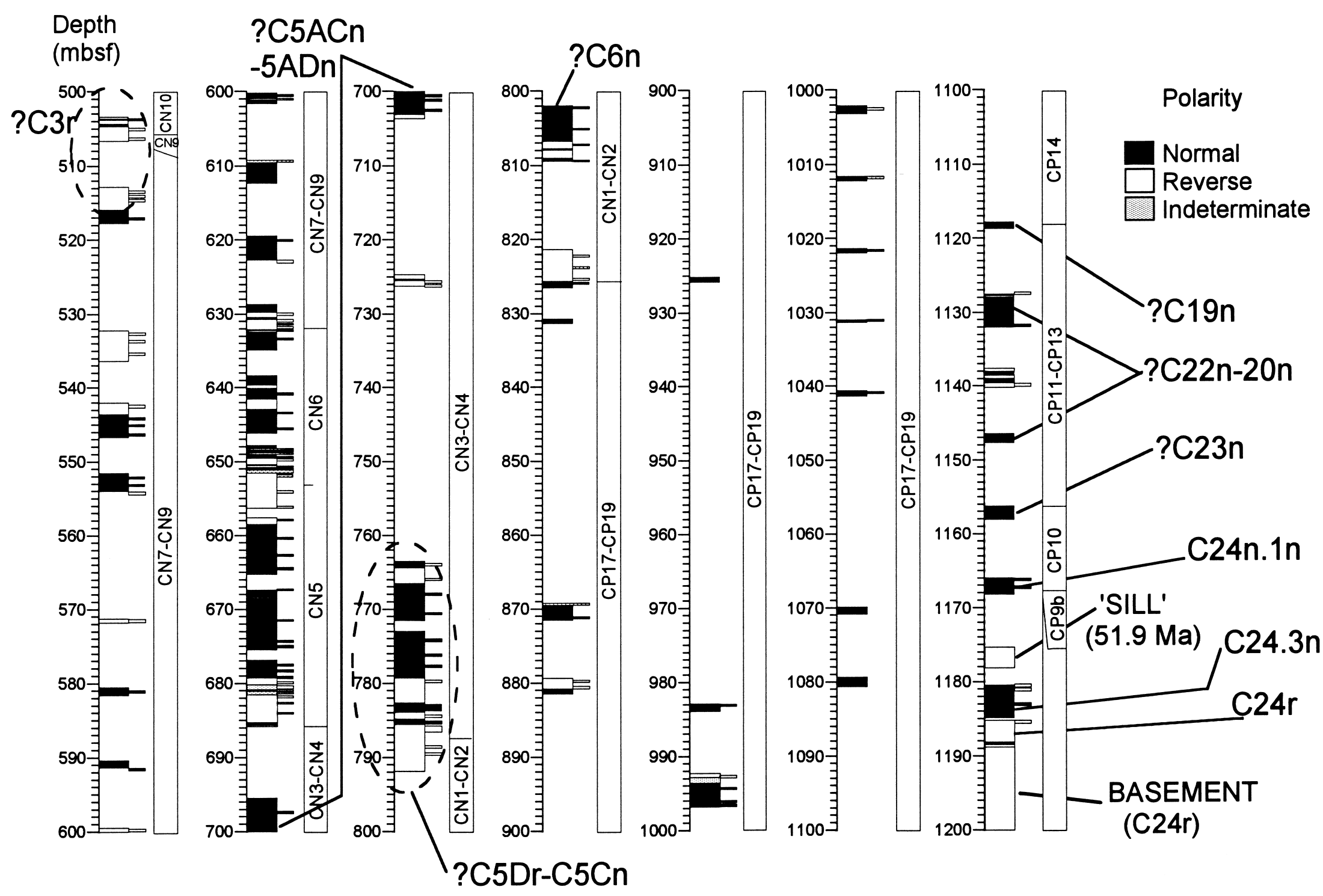

Figure 2. Magnetostratigraphy of Hole 918D, western Irminger Basin. Polarity bars adjacent to the depth scale represent shipboard whole-core determinations. Thin bars to the right of these represent the polarities of discrete specimens. Nannofossil biostratigraphy (CP9b-CN10) based on Wei (this volume). Palynology based on Jolley (this volume). Although all the correlated magnetozones have queries associated with them, those spanning Chrons $\mathrm{C} 24 \mathrm{r}$ through $\mathrm{C} 19 \mathrm{n}$ are considered reliable. 


\section{SITE 919}

No Eocene-Miocene sediments were drilled at Site 919.

\section{SUMMARY AND CONCLUSIONS}

Sites 914, 916, 917, and 918, drilled on the southeast Greenland Margin and in the western Irminger Basin, recovered EoceneMiocene sediments suitable for magnetostratigraphic studies. Wholecore and discrete specimen data have been integrated with biostratigraphic data, and attempts have been made to link the records to the GPTS. Incomplete recovery and limited biostratigraphic information at a number of levels have made this task difficult. Probably the most useful information is provided by Hole 918D, where the magnetobiostratigraphic record from sediments immediately above basement record the early history of the western Irminger Basin (early to middle Eocene). Sedimentation at Site 918, central in the SDRS, commenced very shortly after SDRS formation at $\sim 54-53.5 \mathrm{Ma}$. Sedimentation across the landward edge of the SDRS at the southeast Greenland ocean/continent transition appears to have started much later (13 m.y. for Site 915 and 4 m.y. for Site 917). This prolonged sedimentation time lag between Sites 917 and 915, only $\sim 3 \mathrm{~km}$ apart, probably reflects the complexities of the local basement structure induced during rupture at the ocean/continent transition.

Magnetobiostratigraphic correlations for the younger part of the sequence in Hole 918D place some constraints on Arctic-North Atlantic overflow (11-12 Ma) and southeast Greenland glaciation (at least pre Chron C3r, 5.89 Ma). Unfortunately, our objective of linking more closely North Atlantic faunal and magnetic polarity records has been unsuccessful because of the poor recovery in the lower part of Hole 918D.

\section{ACKNOWLEDGMENTS}

NERC funded JRA's participation on ODP Leg 152 and three months post-cruise studies in the Southampton University Paleomagnetism Laboratory. Ernie Hailwood (Core Magnetics Ltd) kindly reviewed an earlier draft of this manuscript. Mike Fuller, Wuchang Wei, Hans Christian Larsen, and Bob Wathen are thanked for their valuable reviews.

\section{REFERENCES}

Cande, S.C., and Kent, D.V., 1992. A new geomagnetic polarity time scale for the Late Cretaceous and Cenozoic. J. Geophys. Res., 97:1391713951.

, 1995. Revised calibration of the geomagnetic polarity time scale for the Late Cretaceous and Cenozoic. J. Geophys. Res., 100:6093-6095. Larsen, H.C., Saunders, A.D., Clift, P.D., et al., 1994. Proc. ODP, Init. Repts., 152: College Station, TX (Ocean Drilling Program).

Zijderveld, J.D.A., 1967. AC demagnetization of rocks: analysis of results. In Collinson, D.W., Creer, K.M., and Runcorn, S.K. (Eds.), Methods in Palaeomagnetism: New York (Elsevier), 254-286.

Date of initial receipt: 31 July 1995

Date of acceptance: 23 February 1996 Ms 152SR-222 